\title{
AN EXTENSION OF THE CLOSED UNBOUNDED FILTER
}

\author{
ROBERT J. MIGNONE
}

(Communicated by Thomas J. Jech)

\begin{abstract}
A natural extension of the closed unbounded filter is introduced. This extension coincides with the closed unbounded filter on uncountable, regular cardinals $\kappa$, but in general does not for $P_{\kappa} \lambda$ and $[\lambda]^{\kappa}$.
\end{abstract}

Henceforth, $\kappa$ will be a regular, uncountable cardinal, unless specified otherwise. A closed unbounded subset of $\kappa$ is a cofinal subset of $\kappa$ which contains the supremum of all increasing sequences from the subset of length less than $\kappa$. The collection of all closed unbounded subsets of $\kappa$ generates a $\kappa$-complete, normal filter over $\kappa$ called the club filter. The notion of a closed unbounded subset of a cardinal $\kappa$ has been generalized to the set of all subsets of $\lambda$ of cardinality less than $\kappa, P_{\kappa} \lambda$ (see [3]), and the set of all subsets of $\lambda$ of cardinality $\kappa,[\lambda]^{\kappa}$ (see [2]). In both instances the collection of closed unbounded sets generate $\kappa$-complete, fine, normal filters, the club filters. This paper introduces a natural extension of the club filter. This extension coincides with the club filter on $\kappa$, but in general does not for $P_{\kappa} \lambda$ and $[\lambda]^{\kappa}$.

The motivation for the filter arose from the desirable property of certain sequences (or proper chains in the case of $\left.P_{\kappa} \lambda\right)\left\{p_{\alpha}: \alpha<\delta\right\}$ which satisfy

$$
\left|\bigcup_{\alpha<\delta} p_{\alpha}\right|=\bigcup_{\alpha<\delta}\left|p_{\alpha}\right|,
$$

where $|A|$ denotes the cardinality of $A$. Since

$$
\left|\bigcup_{\alpha<\delta} p_{\alpha}\right|=|\delta| \bigcup_{\alpha<\delta}\left|p_{\alpha}\right|
$$

this property is satisfied by sequences (or chains) $\left\{p_{\alpha}: \alpha<\delta\right\}$ which satisfy

$$
|\delta| \leq \bigcup_{\alpha<\delta}\left|p_{\alpha}\right|
$$

A canonical example of a sequence which fails to satisfy this is $\left\{p_{\alpha}: \alpha<\delta\right\}$ where $p_{\alpha}=\alpha$ and $\delta=\omega_{1}$.

DEFINITION. A subset " $b$ " of $\kappa$ is said to be $L$-closed if for any sequence $\left\{p_{\alpha}: \alpha<\right.$ $\delta\} \subset b$ with $\delta<\kappa$ and $|\delta| \leq \bigcup_{\alpha<\delta}\left|p_{\alpha}\right|$, then $\bigcup_{\alpha<\delta} p_{\alpha} \in b$.

Received by the editors July 20, 1987. The results contained in this paper were presented by the author during the 93rd Annual Meeting of the AMS, January 1987, San Antonio, Texas.

1980 Mathematics Subject Classification (1985 Revision). Primary 04A10.

Research was partially supported by a College of Charleston Research and Development grant. The author is grateful to the referee for a careful reading, and for simplifications that were suggested and incorporated in the proof of Proposition 3. 
An $L$-closed, unbounded subset of $\kappa$ is a cofinal subset of $\kappa$ which is $L$-closed. The verification that the $L$-closed, unbounded subsets of $\kappa$ generate a $\kappa$-complete, normal filter over $\kappa$, called the $L$-club filter, is a routine exercise once familiar with the details of generating the club filter over $\kappa$ (see [4]). The $L$-club filter and the club filter coincide on $\kappa$ : Let $b \subset \kappa$ be closed unbounded. Then $b$ is $L$-closed unbounded. And if $b \subset \kappa$ is $L$-closed unbounded, consider

(i) if $\kappa$ is a limit cardinal

$$
a=b \cap\{\alpha<\kappa: \alpha \text { is a cardinal }\} .
$$

(ii) if $\kappa$ is a successor cardinal and $\kappa=\gamma^{+}$

$$
a=(b \cap \kappa-\gamma) \text {. }
$$

In both cases $a \subset b$ and $a$ is closed unbounded.

For $X \subset P_{\kappa} \lambda, X$ is unbounded if for any $x \in P_{\kappa} \lambda$, then there exists a $y \in X$ such that $x \subset y$. And $X$ is closed if for any collection $\left\{p_{\alpha}: \alpha<\delta\right\} \subset X$ with $p_{\alpha} \subset p_{\alpha+1}$ for $\alpha<\delta$ (called a chain of subsets from $P_{\kappa} \lambda$ ) with $\delta<\kappa$, then $\bigcup_{\alpha<\delta} p_{\alpha} \in X$.

DEFinition. A set $X \subset P_{\kappa} \lambda$ is said to be $L$-closed if whenever $\left\{p_{\alpha}: \alpha<\delta\right\} \subset X$ is a proper chain such that $|\delta| \leq \bigcup_{\alpha<\delta}\left|p_{\alpha}\right|<\kappa$, then $\bigcup_{\alpha<\delta} p_{\alpha} \in X$.

Let

$$
L_{\kappa} \lambda=\left\{A \subset P_{\kappa} \lambda \text { : there exists an } X \subset A \text { which is } L \text {-closed unbounded }\right\} \text {. }
$$

PROPOSITION 1. $L_{\kappa} \lambda$ is a $\kappa$-complete, fine, normal filter over $P_{\kappa} \lambda$.

PROOF. The proof follows closely the proof that the closed unbounded sets on $P_{\kappa} \lambda$ generate the club filter (see [3]). The only modification for this proof requires that wherever a chain $\left\{p_{\alpha}: \alpha<\delta\right\}$ is constructed in the club filter proof, the chain must be made to satisfy $|\delta| \leq \bigcup_{\alpha<\delta}\left|p_{\alpha}\right|$. This presents little difficulty in any of the situations where chains are needed. The proof of the next proposition will show the ease at which a chain, in most of the circumstances required here, can be made to satisfy this condition.

A subset $D \subset P_{\kappa} \lambda$ is said to be directed if given $x, y \in D$, then there exists $z \in D$ such that $x \cup y \subset z$. A subset $X \subset P_{\kappa} \lambda$ is said to be closed under directed sets if given $D \subset X$ such that $|D|<\kappa$ and $D$ is directed, then $\bigcup D \in X$. It is a result of Solovay that $X$ is closed unbounded if and only if $X$ is closed under directed sets (see [5]). The analog here is the following:

PROPOSITION 2. An L-closed unbounded subset of $P_{\kappa} \lambda$ is closed under unions of directed sets $D$ where $|D| \leq \bigcup\{|p|: p \in D\}$.

PROOF. (This is basically Solovay's proof with the required modifications.) Let $B$ be an $L$-closed unbounded subset of $P_{\kappa} \lambda$ and $D \subset B$ a directed set such that $|D| \leq \bigcup\{|p|: p \in D\}$. Only the case where $|D|>\aleph_{0}$ requires an adjustment. Assume if $D^{\prime}$ is any directed subset of $B,\left|D^{\prime}\right|<|D|$ and $\left|D^{\prime}\right| \leq \bigcup\{|p|: p \in D\}$, then $\cup D^{\prime} \in B$.

Claim. Given any set $X \subset D$, there exists a set $X^{+}$such that

(1) $X \subset X^{+} \subset D$,

(2) $\left|X^{+}\right| \leq|X|+\aleph_{0} \leq \bigcup\left\{|p|: p \in X^{+}\right\}$and

(3) $X^{+}$is directed.

Proof of Claim. See [5]. 
Well order $D$ by $p_{0}, p_{1}, \ldots, p_{\alpha}, \ldots$ where $\alpha<|D|$. Let

$$
\begin{aligned}
D_{0} & =\left\{p_{0}\right\} \\
D_{1} & =\left\{D_{0}, p_{1}\right\}^{+}, \\
D_{2} & =\left\{D_{0}, D_{1}, p_{2}, q_{2}\right\}^{+} \text {where } q_{2} \in D \text { such that }\left|q_{2}\right| \geq 2, \\
& \vdots \\
D_{\alpha} & =\left\{\left\{D_{\beta}\right\}_{\beta<\alpha}, p_{\alpha}, q_{\alpha}\right\}^{+},
\end{aligned}
$$

where $q_{\alpha} \in D$ such that $\left|q_{\alpha}\right| \geq \alpha+\aleph_{0}$. (Note: such a $q_{\alpha}$ exists since $|D| \leq$ $\bigcup\{|p|: p \in D\}$.) Now,

$$
\left|D_{\alpha}\right|=\left|\left\{\left\{D_{\beta}\right\}_{\beta<\alpha}, p_{\alpha}, q_{\alpha}\right\}^{+}\right| \leq \alpha+\aleph_{0}<|D| .
$$

Hence,

$$
\left|D_{\alpha}\right| \leq \bigcup\left\{|p|: p \in D_{\alpha}\right\}
$$

By our assumption, $\bigcup D_{\alpha} \in B$. So let

$$
Q_{\alpha}=\bigcup D_{\alpha} \in B \quad \text { for all } \alpha<|D| .
$$

Then $\left\{Q_{\alpha}: \alpha<|D|\right\} \subset B$ is a chain and

$$
\begin{aligned}
|D| & \leq \bigcup\{\alpha+: \alpha<|D|\} \leq \bigcup\left\{\left|q_{\alpha}\right|: \alpha<|D|\right\} \\
& \leq \bigcup\left\{\left|U D_{\alpha}\right|: \alpha<|D|\right\}=\bigcup\left\{\left|Q_{\alpha}\right|: \alpha<|D|\right\} .
\end{aligned}
$$

Since $B$ is $L$-closed, $\bigcup D=\bigcup_{\alpha<|D|} Q_{\alpha} \in B$.

In contrast to the situation on cardinals where the $L$-club and club filters coincide, the next proposition shows that in general this not the case for $P_{\kappa} \lambda$ when a large cardinal assumption is made on $\kappa$.

PROPOSITION 3. Assume $\kappa$ is a huge cardinal and $\lambda$ is any cardinal greater than $\kappa$ such that there exists a $\kappa$-complete, fine, normal ultrafilter over $[\lambda]^{\kappa}$, then there exists an L-closed unbounded subset of $P_{\kappa} \lambda$ which is not in the club filter.

Proof. Since $\lambda$ must be measurable (see [1 or 6]), a regular cardinal $\gamma$ can be chosen such that $\kappa<\gamma<\lambda$. Now $\lambda$ must be a regular cardinal so $\lambda$ can be partitioned into $\lambda$-many disjoint intervals of length $\gamma$. For $\alpha<\lambda$ denote the $\alpha$ th such interval as $\gamma_{\alpha}$. Given any $\beta<\lambda$ let the $\gamma$ th index of $\beta$, denoted $\gamma(\beta)$, be

$$
\Gamma(\beta)=\text { order type of } \gamma_{\alpha} \cap \beta, \quad \text { where } \beta \in \gamma_{\alpha} \text {. }
$$

For $x \in P_{\kappa} \lambda$ let

$$
\Gamma^{\prime \prime} x=\{\Gamma(\beta): \beta \in x\}
$$

Set

$$
B=\left\{x \in P_{\kappa} \lambda:\left|\Gamma^{\prime \prime} x\right|=|x|\right\} .
$$

Claim 1. $B$ is $L$-closed unbounded.

ProOF OF CLAIM. $B$ is readily seen to be unbounded, since for any $x \in P_{\kappa} \lambda$, $x \cup|x| \cup \omega \in B$.

Next, let $\left\{p_{\alpha}: \alpha<\delta\right\} \subset B$ be a chain of size $\delta<\kappa$, where $|\delta| \leq \bigcup_{\alpha<\delta}\left|p_{\alpha}\right|$. Suppose $\left|\bigcup_{\alpha<\delta} p_{\alpha}\right|>\left|\Gamma^{\prime \prime} \bigcup_{\alpha<\delta} p_{\alpha}\right|$. Let $\beta=\left|\Gamma^{\prime \prime} \bigcup_{\alpha<\delta} p_{\alpha}\right|$. So $\left|\bigcup_{\alpha<\delta} p_{\alpha}\right|>\beta$. 
Since $\bigcup_{\alpha<\delta}\left|p_{\alpha}\right|=\left|\bigcup_{\alpha<\delta} p_{\alpha}\right|>\beta$ there exists $\alpha<\delta$ such that $\left|p_{\alpha}\right|>\beta$. But $\left|\Gamma^{\prime \prime} p_{\alpha}\right|=\left|p_{\alpha}\right|$, which is clearly false. Therefore, $\bigcup_{\alpha<\gamma} p_{\alpha} \in B$, and the claim is proved.

DiPrisco and Marek used the following type of construction to define their notion of closed unbounded sets on $[\lambda]^{\kappa}$ (see [2]):

For $B \subset P_{\kappa} \lambda$ from above, set

$$
A_{B}=\left\{p \in[\lambda]^{\kappa}: \text { there exists a chain }\left\{p_{\alpha}: \alpha<\kappa\right\} \subset B \text { and } p=\bigcup_{\alpha<\kappa} p_{\alpha}\right\} \text {. }
$$

CLAIM 2. If $p \in A_{B}$, then $\left|\Gamma^{\prime \prime} p\right|=\kappa$.

ProOF OF ClaIM. Assume $\left|\Gamma^{\prime \prime} p\right|<\kappa$. Since $\kappa=|p|=\left|\bigcup_{\alpha<\kappa} p_{\alpha}\right|=\bigcup_{\alpha<\kappa}\left|p_{\alpha}\right|$ there exists $\alpha<\kappa$ such that $\left|p_{\alpha}\right|>\left|\Gamma^{\prime \prime} p\right|$. But this gives

$$
\left|\Gamma^{\prime \prime} p_{\alpha}\right|=\left|p_{\alpha}\right|>\left|\Gamma^{\prime \prime} p\right|=\left|\Gamma^{\prime \prime} \bigcup_{\alpha<\kappa} p_{\alpha}\right|
$$

which cannot be true.

Finally, assume there exists a closed unbounded set $C$ from $P_{\kappa} \lambda$ which is a subset of $B$. By a result in [2] if $U$ is any $\kappa$-complete, fine, normal ultrafilter over $[\lambda]^{\kappa}$ and $C$ is any closed unbounded subset of $P_{\kappa} \lambda$, then $A_{C} \in U$. So by our assumption, $A_{B} \in U$. That is, $\left\{p \in[\lambda]^{\kappa}:\left|\Gamma^{\prime \prime} p\right|=\kappa\right\} \in U$.

Let $j: V \rightarrow M$ be the canonical elementary embedding produced by the ultrafilter $U$ on $[\lambda]^{\kappa}$. Then, $A \in U$ iff $j^{\prime \prime} \lambda \in j(A)$. Hence,

$$
M \vDash(j \Gamma)^{\prime \prime} j^{\prime \prime} \lambda=j(\kappa)
$$

by the above. However, the definition of $\Gamma$ and the elementarity of $j$ yield

$$
M \vDash(j \Gamma)^{\prime \prime} j^{\prime \prime} \lambda=j^{\prime \prime} \gamma .
$$

But, this is a contradiction, since $\gamma<\lambda$.

This demonstrates that there are subsets of $P_{\kappa} \lambda$ which are $L$-closed unbounded but not closed. However, such subsets of $P_{\kappa} \kappa^{+}$do not exist.

Whether or not an $L$-closed unbounded subset of $P_{\kappa} \lambda$ can be constructed which does not contain a closed unbounded subset, without first assuming the existence of a huge cardinal, is open. However, there exist examples of $L$-closed unbounded subsets of $P_{\kappa} \lambda$ which are not closed unbounded. The following was provided by C. A. DiPrisco:

$$
E=\left\{p \in P_{\kappa} \lambda:|p \cap \kappa|=|p-\kappa|\right\} .
$$

The problem remains to determine whether or not such sets are in the club filter on $P_{\kappa} \lambda$.

\section{REFERENCES}

1. J. B. Barbanel, C. A. DiPrisco and I. B. Tan, Many-times huge and supercompact cardinals, J. Symbolic Logic 49 (1984), 177-187.

2. C. A. DiPrisco and W. Marek, A filter on $[\lambda]^{\kappa}$, Proc. Amer. Math. Soc. 90 (1984), 591-598.

3. T. J. Jech, Some combinatorial problems concerning uncountable cardinals, Ann. Math. Logic 5 (1973), 165-198.

4. 
5. M. Magidor, Combinatorial characterizations of supercompact cardinals, Proc. Amer. Math. Soc. 42 (1974), 279-285.

6. R. J. Mignone, The ultrafilter characterization of huge cardinals, Proc. Amer. Math. Soc. 90 (1984), 585-590.

Department of Mathematics, The College of Charleston, Charleston, SOUTH CAROLINA 29424 